\title{
Reptin drives tumour progression and resistance to chemotherapy in nonsmall cell lung cancer
}

\author{
Jan-Henrik Mikesch ${ }^{1}$, Daniela Schwammbach ${ }^{1}$, Wolfgang Hartmann ${ }^{2}$, \\ Lars H. Schmidt ${ }^{1}$, Christoph Schliemann ${ }^{1}$, Linus Angenendt ${ }^{1}$, Rainer Wiewrodt ${ }^{1}$, \\ Alessandro Marra ${ }^{3}$, Nils H. Thoennissen ${ }^{1}$, Eva Wardelmann², Gabriele Köhler ${ }^{2,4}$, \\ Georg Lenz ${ }^{1}$, Carsten Müller-Tidow ${ }^{1,5}$, Wolfgang E. Berdel ${ }^{1}$ and \\ Maria-Francisca Arteaga ${ }^{1}$
}

Affiliations: ${ }^{1}$ Dept of Medicine A, University of Muenster, Muenster, Germany. ${ }^{2}$ Gerhard Domagk Institute for Pathology, University of Muenster, Muenster, Germany. ${ }^{3}$ Dept of Thoracic Surgery, Klinikum Bremen Ost, Bremen, Germany. ${ }^{4}$ Institute of Pathology, Klinikum Fulda, Fulda, Germany. ${ }^{5}$ Dept of Medicine V, University of Heidelberg, Heidelberg, Germany.

Correspondence: Maria-Francisca Arteaga, Dept of Medicine A, Hematology and Oncology, University of Muenster, Albert-Schweitzer Campus, 48149 Muenster, Germany. E-mail: marifrancis.arteagaduni-muenster.de

@ERSpublications

The AAA+ ATPase Reptin confers tumour progression, resistance to chemotherapy and poor outcome in NSCLC patients http://ow.ly/dLxW30kaUVu

Cite this article as: Mikesch J-H, Schwammbach D, Hartmann W, et al. Reptin drives tumour progression and resistance to chemotherapy in nonsmall cell lung cancer. Eur Respir J 2018; 52: 1701637 [https://doi. org/10.1183/13993003.01637-2017].

ABSTRACT While targeted nonsmall cell lung cancer (NSCLC) therapies have improved the outcome of defined disease subtypes, prognosis for most patients remains poor. We found the AAA+ ATPase Reptin to be highly expressed in the vast majority of 278 NSCLC tumour samples. Thus, the objective of the study was to assess the role of Reptin in NSCLC.

Survival analyses of 1145 NSCLC patients revealed that high RNA expression levels of Reptin are associated with adverse outcome. Knockdown of Reptin in human NSCLC cells impaired growth ex vivo and eliminated engraftment in a xenograft model. Reptin directly interacted with histone deacetylase 1 (HDAC1) as the critical mechanism driving NSCLC tumour progression. Pharmacological disruption of the Reptin/HDAC1 complex resulted in a substantial decrease in NSCLC cell proliferation and induced significant sensitisation to cisplatin.

Our results identify Reptin as a novel independent prognostic factor and as a key regulator mediating proliferation and clonal growth of human NSCLC cells ex vivo and in vivo. We unveil a Reptin/HDAC1 protein complex whose pharmacological disruption sensitises NSCLC cells to cisplatin, suggesting this approach for application in clinical trials. 


\section{Introduction}

The identification of various oncogenic driver mutations [1] and evidence of high expression of distinct membrane proteins modulating the immune system have enabled the development of powerful targeted nonsmall cell lung cancer (NSCLC) therapies [2-4]. In spite of these breakthrough discoveries, responses to most standard NSCLC therapies are still poor and/or short-lived. To date, lung cancer represents the leading cause of cancer mortality worldwide, and the prognosis especially of NSCLC patients with advanced or metastatic disease remains poor [5]. Determining parameters that predict early metastasis or dismal prognosis may help to identify patients requiring alternative treatment strategies. Moreover, the molecular mechanisms underlying carcinogenesis and tumour progression need to be unravelled so that more specific and efficacious substances targeting NSCLC can be designed.

NSCLC is largely determined by transcriptional deregulation based on a broad variety of genetic and epigenetic events. Alterations of the transcriptional programmes result in cellular transformation and/or tumour progression and maintenance [6]. In the last few years, the AAA+ ATPase family member Reptin has been implicated in various physiological processes, including transcriptional regulation, remodelling of chromatin and repair of DNA damage [7, 8], mechanisms that are frequently deteriorated in NSCLC cells. Thus, we sought to determine the role of Reptin in lung cancer and as a potential novel target for future NSCLC therapies.

\section{Material and methods}

Lung cancer tissue microarray

Tumour material and clinical follow-up data were available from 321 NSCLC patients from the thoracic departments in Ostercappeln, Germany (study collective I; $n=265$ NSCLC tissue samples) and Mainz, Germany (study collective II; $\mathrm{n}=56$ NSCLC tissue samples) (median age 66 years) who underwent curative resection. Clinical follow-up information and study approval by the ethical committees of Muenster and Mainz were obtained for the collection of paraffin-embedded tissue samples for biomarker testing. Due to the retrospective, anonymous character of the analysis, written patient consent was not required. Clinical TNM staging (including clinical examination, computed tomography scans, sonography, endoscopy, magnetic resonance imaging and bone scan) was based on Union for International Cancer Control/ American Joint Committee on Cancer recommendations. In terms of definite tumour staging, pathological exploration was carried out after surgery. Primary pulmonary lesions were pathologically classified based on the World Health Organization 2004 guidelines; 149 specimens were classified as squamous cell carcinoma (SCC), 125 as adenocarcinoma (AC) and 47 as large cell carcinoma. Patients with stage IV, neoadjuvant treatment, R1 or R2 resection status or with non-specified tumour histology (e.g. NSCLC not otherwise specified) were excluded from our study. Thus, 278 patient samples remained for analysis. Regular follow-up was performed for all patients, including systemic re-staging after 3, 6, 12, 18, 24, 36, 48 months etc. or earlier, if clinically required. Survival time was either computed from the date of histological diagnosis to death or to the date of last contact. Baseline information of the NSCLC population is shown in supplementary table S1. Tissue microarrays (TMAs) were generated from formalin-fixed, paraffin-embedded tissue specimens and as described previously [9]. The following primary antibody was applied: Anti-human Reptin antibody (ab no. 612482, clone 42/TIP49b; BD Biosciences, Heidelberg, Germany). Immunoreaction was visualised with a biotinylated secondary antibody (LSAB/AP, \#K5005; Dako, Glostrup, Denmark) including the red chromogen and according to the manufacturer's instructions. Immunohistochemical assessment was performed by applying a quantitative $\mathrm{H}$-score as a continuous value, essentially based on staining intensity according to parameters proposed by Harvey et al. [10], as well as percentage of stained cells. Prototypical examples of four different staining intensities $(0=$ none, $1=$ weak, $2=$ moderate, 3 =high) were assembled through an initial screen of the stained set of samples to establish a reference framework (supplementary methods). The staining intensity of each tumour sample was determined based on the reference framework. Proportional information was integrated through the assignment of heterogeneously staining tumours to the higher intensity category if at least $10 \%$ of the tumour cells fulfilled its criteria. Four investigators (J.-H.M., W.H., G.K., M.F.A.) independently analysed TMA slides. Samples with discordant assessment results were re-evaluated and a consensus was reached. The $\mathrm{H}$-score was calculated as intensity $\times \%$ stained cells. Optimal cut-off for overall survival was assessed using maximally selected log-rank statistics in $\mathrm{R}$ version 3.4.3. The association of clinico-pathological parameters with Reptin expression was tested using two-sided Fisher's exact test. Univariate overall survival analysis was performed using the Kaplan-Meier method and log-rank tests. A multivariable Cox proportional hazards model was fitted using a forward step-wise variable selection (inclusion criteria: p-value of the likelihood ratio test $\leqslant 0.05$ ) to identify independent prognostic factors for overall survival. Patients with missing values in the cofactors were excluded from the analysis. All statistical tests were performed as exploratory analyses on a local significance level of 0.05 . SPSS (SPSS Statistics, Version 22.0 released 2013, IBM Corp., Armonk, NY, USA) was used for all statistical analyses. 
Plasmids, antibodies and primers

Human Reptin short hairpin RNAs (shRNAs) were a kind gift from C. Abraham (University of Colorado at Boulder, Boulder, CO, USA). The sequences were: Reptin TRCN0000051563, pLKO shRNA \#1: CCGTGATGTAACAAGGATTGA; and TRCN0000051564, pLKO shRNA \#2: CGAGAAAGACACGAAGC AGAT [11]. Sequences of single guide RNAs (sgRNAs) targeting Reptin are provided in supplementary figure S2e.

HDAC1-pcDNA3 plasmid was a kind gift from K. Glaser (Abbott Laboratories, Chicago, IL, USA). We used the following antibodies in this study: Flag M2 (A8592) and anti- $\beta$-actin (A5441; both Sigma-Aldrich, Saint Louis, MO, USA); Reptin antibody (anti-TIP49b; 612482; BD Biosciences, Heidelberg, Germany); anti-CK7 (SP52) rabbit monoclonal and OptiView DAB IHC Detection Kit (790-4462; both Ventana Medical Systems, Oro Valley, AZ, USA); and goat anti-mouse (115-036-062) and goat anti-rabbit (111-036-045; both Dianova, Hamburg, Germany).

\section{Cell culture and reagents}

Human NSCLC cell lines A549, LUDLU 1 and HOP62 were obtained from Sigma-Aldrich (Salisbury, UK) and Charles River Laboratories (Cologne, Germany), respectively.

A549 and HOP62 cells were maintained in D10 medium (DMEM, 10\% fetal calf serum (FCS), $100 \mathrm{U} \cdot \mathrm{mL}^{-1}$ penicillin and $100 \mu \mathrm{g} \cdot \mathrm{mL}^{-1}$ streptomycin). LUDLU 1 cells were maintained in R10 medium (RPMI, 10\% FCS, $100 \mathrm{U} \cdot \mathrm{mL}^{-1}$ penicillin and $100 \mu \mathrm{g} \cdot \mathrm{mL}^{-1}$ streptomycin). The DMEM (D5796), RPMI (R8758), penicillin/streptomycin (P0781-100ML) and INT p-Iodonitrotetrazolium Violet ((INT) I8377) were obtained from Sigma-Aldrich (Saint Louis, MO, USA). FCS (S0115) was obtained from Biochrom (Cambridge, UK) and trypsin (BE02-007E) from Lonza (Basel, Switzerland). Entinostat (MS-275, sc-279455) came from Santa Cruz Biotechnology (Santa Cruz, CA, USA), cisplatin (PZN 06559665) from TEVA (Ulm, Germany) and dimethyl sulfoxide ((DMSO) 20385.01) from SERVA Electrophoresis GmbH (Heidelberg, Germany).

Lentiviral transduction, colony formation and proliferation assays

Lentiviral transduction was performed on NSCLC cells, adding $6 \mathrm{~mL}$ of D10 containing virus and $6 \mu \mathrm{g} \cdot \mathrm{mL}^{-1}$ polybrene (107689; Sigma, Saint Louis, MO, USA). Transduced cells were selected using appropriate antibiotics.

To knock down human Reptin, cells were transduced with pLKO.1 puromycin plasmids containing shRNAs targeting human Reptin. Scrambled sequences were used as controls (sequences are available upon request). After antibiotic selection, cells were plated into methylcellulose medium containing specific drug treatment. Colonies were scored after 7-10 days of culture and labelled with INT overnight.

Proliferation assays were performed using CellTiter $96^{\circ}$ Aqueous Non-Radioactive Cell Proliferation Assay (G54301; Promega, Madison, WI, USA).

\section{Immunoprecipitation and immunoblot analysis}

For generic immunoprecipitation, cells were lysed in NP-40 lysis buffer (0.5\% NP-40, $50 \mathrm{mM}$ Tris-base $\mathrm{pH}$ 7.5, $5 \mathrm{mM}$ EDTA, $150 \mathrm{mM} \mathrm{NaCl}, 0.2 \mu \mathrm{M}$ dithiothreitol, $10 \%$ glycerol, protease inhibitors) for $1 \mathrm{~h}$ at $4^{\circ} \mathrm{C}$. Protein concentration was measured by the PerkinElmer VictorX3 spectrophotometer (Baesweiler, Germany) using the Bio-Rad protein assay reagent (Pierce, Rockford, IL, USA). Equal amounts of total protein were incubated with the respective antibody overnight, precipitated with protein A/G Dynal beads (Invitrogen, Carlsbad, CA, USA) at $4^{\circ} \mathrm{C}$ for $1 \mathrm{~h}$ and then the Dynal beads were washed with $0.5 \%$ NP-40 washing buffer. Eluted proteins were resolved by SDS-PAGE. Membranes were probed with antibodies described above. For immunoblotting, samples were lysed with radioimmunoprecipitation assay buffer $\left(150 \mathrm{mmol} \cdot \mathrm{L}^{-1} \mathrm{NaCl}, 1 \%\right.$ Triton-X-100, 0.5\% Na-deoxycholate, $0.1 \%$ SDS, $50 \mathrm{mmol} \cdot \mathrm{L}^{-1}$ Tris $\cdot \mathrm{HCl}, \mathrm{pH} 8.0$ ). Equal amount of proteins were resolved in NuPAGE Novex 4-12\% Bis-Tris Gel $1.0 \mathrm{~mm}, 10$-well (NP0321Box; Invitrogen, Carlsbad, CA, USA). Polyvinylidene fluoride membranes (Merck Millipore, Burlington, MA, USA) were blotted with indicated antibodies and detected by Western Lightning ECL reagent (PerkinElmer, Baesweiler, Germany). Density quantification of the bands was analysed using Image J software.

\section{Mouse xenograft}

NOD.Cg-Prkdcscid IL2rgtmWjl/Sz (NSG) mice were used for transplantation experiments. Mice were injected intravenously with $1 \times 10^{6}$ test cells. All experimental procedures were approved by the "Landesamt für Natur, Umwelt und Verbraucherschutz NRW" (GEZ 84-02.04.2014.A508) and performed in accordance with the regulations. 
Flow cytometric analysis of human cell lines

Immunophenotypic analysis was performed by flow cytometric analysis using fluorochrome-conjugated monoclonal antibodies, PE anti-human CD326 (epithelial cell adhesion molecule) (mouse IgG2b; 324205; BD Biosciences, Heidelberg, Germany) and PE Mouse IgG1k (isotype control; 555749; BD Biosciences, Heidelberg, Germany).

Staining was generally performed in suspension medium (SM) (PBS, 0.2\% FCS) and incubated on ice for $30 \mathrm{~min}$, washed twice and resuspended in SM before analysis using a BD LSR II system (BD Biosciences, Heidelberg, Germany).

\section{Results}

Reptin is overexpressed in the vast majority of NSCLC tumour tissues

To assess the role of Reptin in NSCLC, we analysed protein expression in tissue samples from 49 NSCLC patients by western blot and from 278 NSCLC patients by immunohistochemistry. Western blot analyses showed that Reptin is significantly overexpressed in tumour tissues versus adjacent normal lung tissues in the vast majority of NSCLC patients (figure 1a, b). Reptin expression was significantly higher in tumour tissues of patients with both pulmonary SCC and non-SCC. There was higher expression of Reptin in the tumour than in the adjacent normal tissue in $92 \%$ of SCC patients $(n=26)$ and $65 \%$ of non-SCC patients

a)

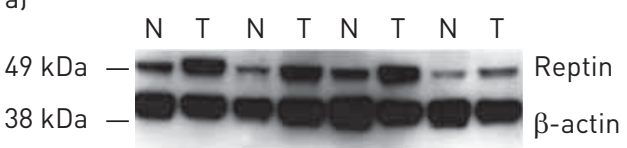

non-SCC
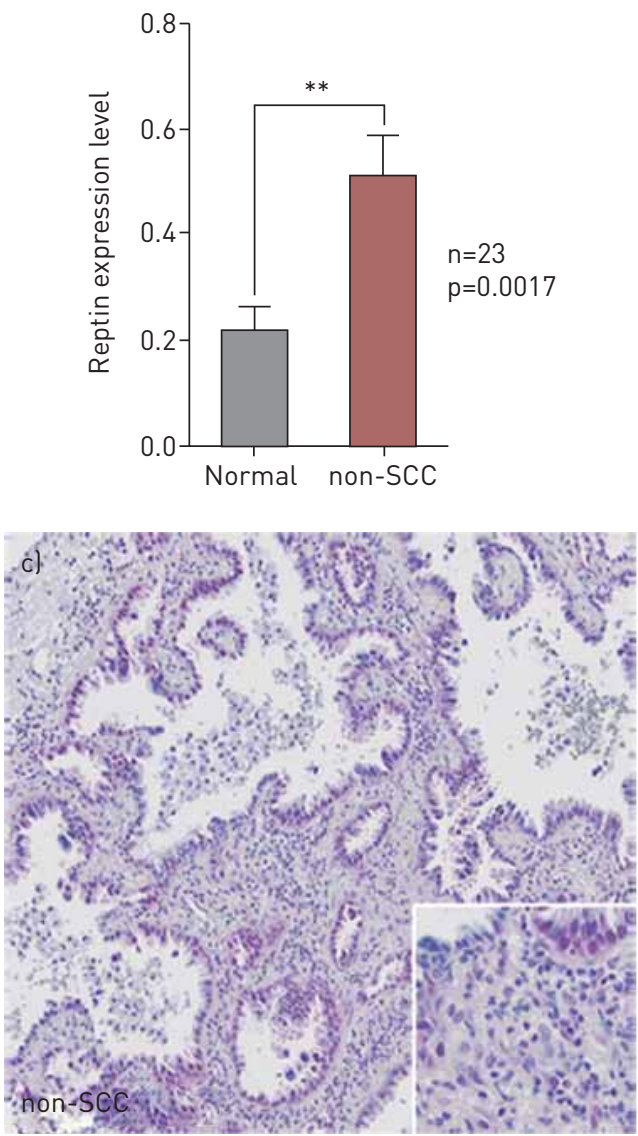

b)

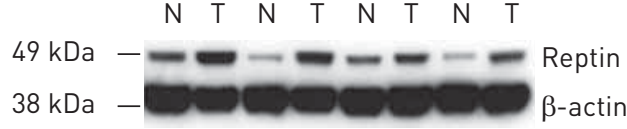

SCC

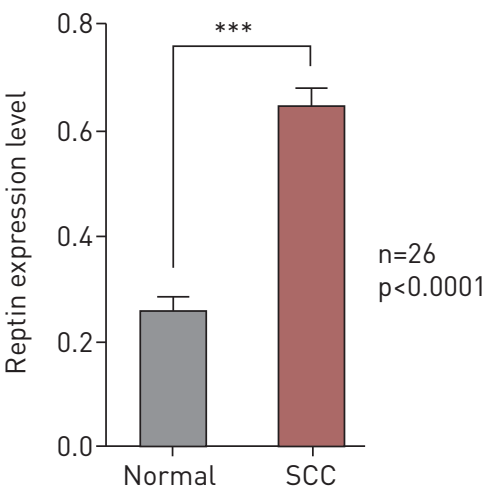

FIGURE 1 Reptin is highly expressed in the majority of human nonsmall cell lung cancer (NSCLC). a, b) Immunoblot analyses and quantification of Reptin expression in tumour tissue (T) versus adjacent normal lung tissue $(\mathrm{N})$ of patients with pulmonary a) non-squamous cell carcinoma (non-SCC) and b) SCC. Error bars represent SD of at least three independent experiments. c, d) Immunohistochemical staining of Reptin in c) non-SCC and d) SCC NSCLC tumour tissues. ${ }^{* *}$ : $p<0.01 ;{ }^{* * *}$ : $p<0.001$. 
$(n=23)$ (figure 1a, b). On immunohistochemistry, tumour cells displayed a nuclear staining pattern for Reptin in both non-SCC and SCC lung cancer tumours (figure 1c, d).

Reptin confers poor prognosis in NSCLC patients

To determine whether overexpression of Reptin affects the prognosis of NSCLC patients, we performed immunohistochemistry on TMA samples from 278 NSCLC patients, including 150 patients with pulmonary non-SCC and 128 patients with SCC (supplementary table S1).

Tumour tissues with low Reptin expression were defined by an H-score $\leqslant 160$ and high Reptin expression by an $\mathrm{H}$-score $>160$. Patients with low Reptin expression showed significantly better overall survival $(\mathrm{p}=0.002)$ (figure 2a). As a validation cohort, we used the survival data of 1145 NSCLC patients from a publicly available dataset [12] to confirm significantly decreased overall survival $(p<0.0001)$ of patients with high mRNA expression levels of Reptin compared with that of patients with low levels (dichotomised at median) (figure 2b, c). Among 596 patients with recorded clinical progression data, time to progression was also significantly reduced for patients with high expression of Reptin ( $p=0.00065$ ) (figure $2 b, c$ ). Multivariate analyses using the same database confirmed Reptin as a risk factor for poor overall and progression-free survival independent of histology and disease stage $(p<0.0001$ and $p=0.0089$, respectively).

Patients with highly differentiated NSCLC tumours (G1-G2) are known to have improved long-term outcomes [13]. Accordingly, in our cohort of NSCLC tumour samples analysed by immunohistochemistry, we were able to confirm a strong trend towards better overall survival for patients with G1-G2 tumours $(n=102)$ than for patients with less differentiated (G3-G4) tumours ( $n=170 ; p=0.063)$ (supplementary figure S1a). Patients with highly differentiated tumours showing high Reptin expression had significantly worse overall survival compared to patients from the same subgroup with low Reptin expression $(\mathrm{p}<0.001)$ (figure $2 \mathrm{~d}, \mathrm{e}$ ). This difference was not seen among patients with poorly differentiated tumours (figure $2 \mathrm{~d}$, e and supplementary figure S1b,c). We confirmed significantly improved overall survival among patients with highly differentiated NSCLC showing low Reptin expression for non-SCC patients $(\mathrm{p}<0.001)$. SCC patients with G1-G2 tumours with high Reptin expression represented a much smaller subgroup in our cohort and yet showed a strong trend towards shorter overall survival $(p=0.086)$ (figure $2 \mathrm{f}, \mathrm{g})$. Thus, survival analyses revealed a significant prognostic impact of high expression of Reptin in NSCLC patients.

\section{Knockdown of Reptin impairs growth of human NSCLC cells ex vivo and blocks their engraftment in vivo}

In order to assess the role of Reptin in growth and proliferation of NSCLC cells, we performed lentiviral transduction with two validated shRNAs targeting human Reptin [11] in A549 and HOP62 human AC cells as well as in LUDLU 1 human pulmonary SCC cells. Stable knockdown of Reptin was achieved in all cell lines as compared to scrambled controls (figure 3a). Suppression of Reptin significantly impaired clonal expansion of all cell lines as assessed by colony numbers after plating in methylcellulose (figure $3 \mathrm{~b}-\mathrm{d}$ ). Moreover, all three cell lines showed a significant reduction in proliferation upon knockdown of Reptin (figure $3 \mathrm{e}-\mathrm{g}$ ). To exclude shRNA-mediated off-target effects we designed sgRNAs targeting different exons of human Reptin (supplementary figure S2e) and cloned them into a lentiviral CRISPR-Cas9 vector. Again, a stable knockdown of Reptin in A549, HOP62 and LUDLU 1 cells was achieved via lentiviral transduction of our constructs (supplementary figure S2a and data not shown). We confirmed dependence of colony formation capacity and proliferation of Reptin expression levels in both pulmonary AC and SCC cells (supplementary figure S2b-d and data not shown).

Knockdown of Reptin in A549 cells was also able to block engraftment in five out of six NSG mice, whereas all mice transplanted with the same cells transduced with a scrambled control developed metastatic lung cancer $(n=6)$ within $70-110$ days (figure $3 \mathrm{~h}$ ). Flow cytometry detected strong expression of human epithelial cell adhesion molecule CD326 in cells isolated from metastatic lesions, demonstrating that organs of diseased mice were heavily infiltrated by human NSCLC cells (figure 3i). Furthermore, histological analyses confirmed infiltration of murine organs by human invasive AC with strong expression of human CK7 (supplementary figure S3a, b). Thus, growth of human NSCLC cells ex vivo and engraftment in vivo substantially depend on the presence of Reptin.

\section{Pharmacological disruption of a Reptin/HDAC1 complex in NSCLC cells significantly reduces clonal growth}

We were able to confirm that Reptin directly interacts with HDAC1, one of its suspected partners for transcriptional repression, in human HEK-293 cells (supplementary figure S4) [14]. In pulmonary AC, expression of HDAC1 correlates with poor patient outcome [15]. To evaluate whether Reptin and HDAC1 also associate in NSCLC cells, we performed further co-immunoprecipitation experiments. We were able to 

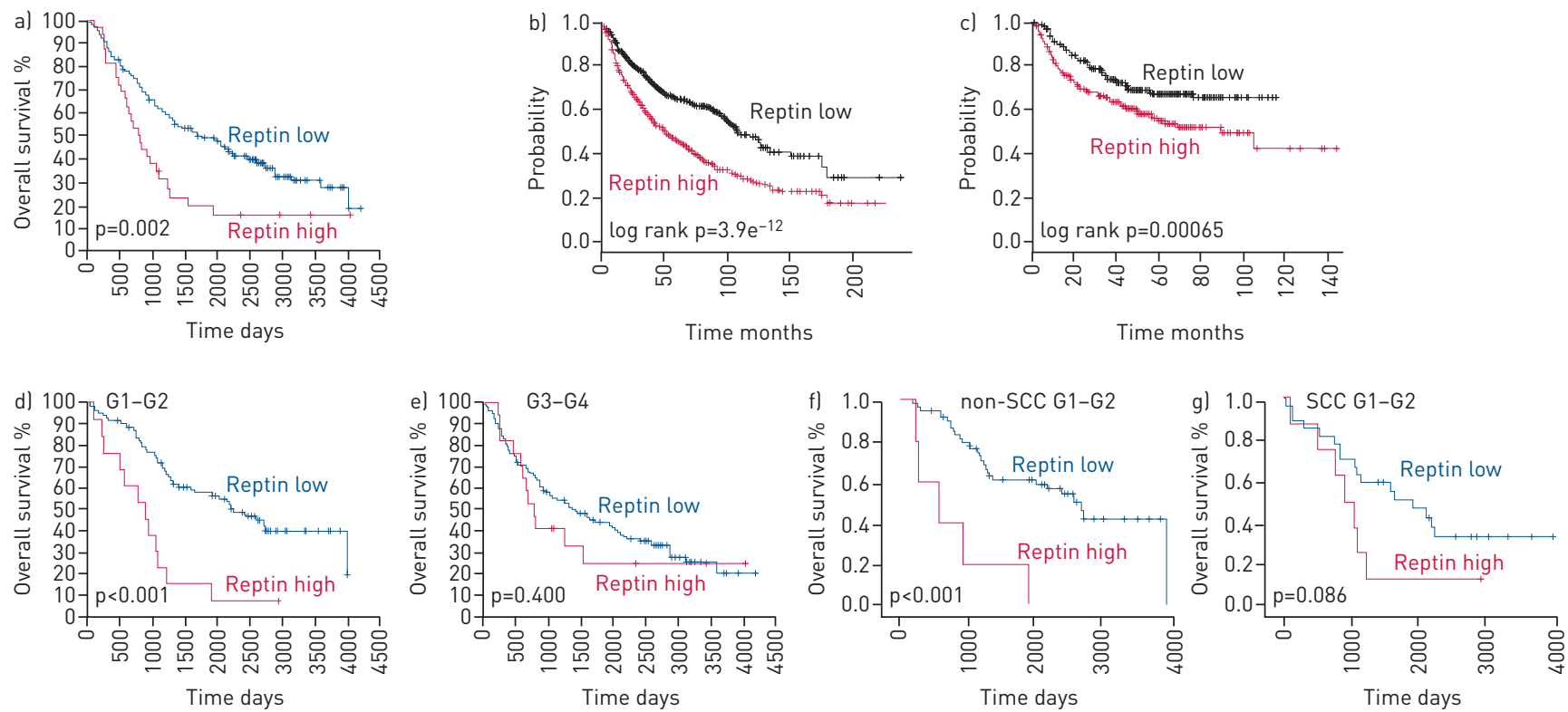

FIGURE 2 Prognostic values of Reptin expression showing that Reptin confers poor prognosis in nonsmall cell lung cancer (NSCLC) patients. a) Overall survival of all patients. b) Overall and c) progression-free survival of NSCLC patients in a larger patient cohort from a publicly available dataset. d, e) Overall survival of NSCLC patients with highly differentiated (G1-2) (d) and poorly differentiated (G3-4) (e) tumours. f, g) Overall survival in non-squamous cell carcinoma (non-SCC) patients with G1-2 tumours ( $f$ ) and SCC patients with G1-2 tumours (g). Unpaired t-test was used to determine statistical significance of the probability of overall and progression-free survival.

detect direct interaction between Reptin and HDAC1 in all three lung cancer cell lines tested (A549, HOP62, LUDLU 1). Interestingly, when we exposed NSCLC cells to MS-275 (entinostat), a class 1 HDAC inhibitor with a special affinity for HDAC1, this protein-protein interaction was significantly reduced in both pulmonary AC and SCC cells (figure 4a). As a next step, we treated A549, HOP62 and LUDLU 1 cells with different concentrations of MS-275. Both proliferation and the colony formation capacity of all three cell lines were significantly reduced upon increased exposure to MS-275 (figure 4b-h). Thus, pharmacological inhibition of the Reptin/HDAC1 protein complex might be a promising new strategy for future therapeutic approaches in NSCLC therapies.

\section{Disrupting the Reptin/HDAC1 complex enhances sensitivity of NSCLC cells to cisplatin}

To date, platinum-doublet chemotherapy remains the backbone for most NSCLC patients in (neo)adjuvant and palliative care settings. Unfortunately, the majority of NSCLC patients either show upfront platinum-resistance or will develop relapse or disease progression after initial treatment response. Thus, we assessed whether combining cisplatin with MS-275 could enhance the therapeutic effect of the chemotherapy. Exposure of pulmonary AC and SCC cells to even low doses of MS-275 profoundly increased the effect of cisplatin on these cells. Low doses of cisplatin induced a mild to moderate reduction of both colony formation capacity and proliferation of A549, HOP62 and LUDLU 1 cells; addition of low doses of MS-275 significantly enhanced its effect (figure 5a-i). To confirm the role of Reptin in this complex for sensitisation of lung cancer cells to treatment with cisplatin, we exposed human NSCLC cells with shRNA-mediated knockdown of Reptin to low doses of cisplatin. Again, the effect of the chemotherapy was significantly more profound compared to that of scrambled controls (figure 5j). Thus, targeting the Reptin/HDAC1 complex might be a valid option to improve the effect of future platinum-based chemotherapy regimens in NSCLC patients.

\section{Discussion}

During the past few years, significant progress has been made in the three pillars of NSCLC management: prevention, diagnostics and therapy $[2,16]$. However, with $>1.5$ million deaths per year, lung cancer remains one of the major causes of cancer deaths worldwide [5]. The major hurdles for successful lung cancer treatment comprise diagnosis at an advanced stage of the disease, early relapses and/or metastasis, and poor responses of patients with metastatic lung cancer to the available therapies. Recent clinical trials provided evidence that identifying and targeting molecular alterations in NSCLC can substantially broaden a physician's repertoire for treating lung cancer [2,4]. However, none of the substances available are expected to cure the majority of NSCLC patients diagnosed with advanced stage of the disease. Thus, 
a)
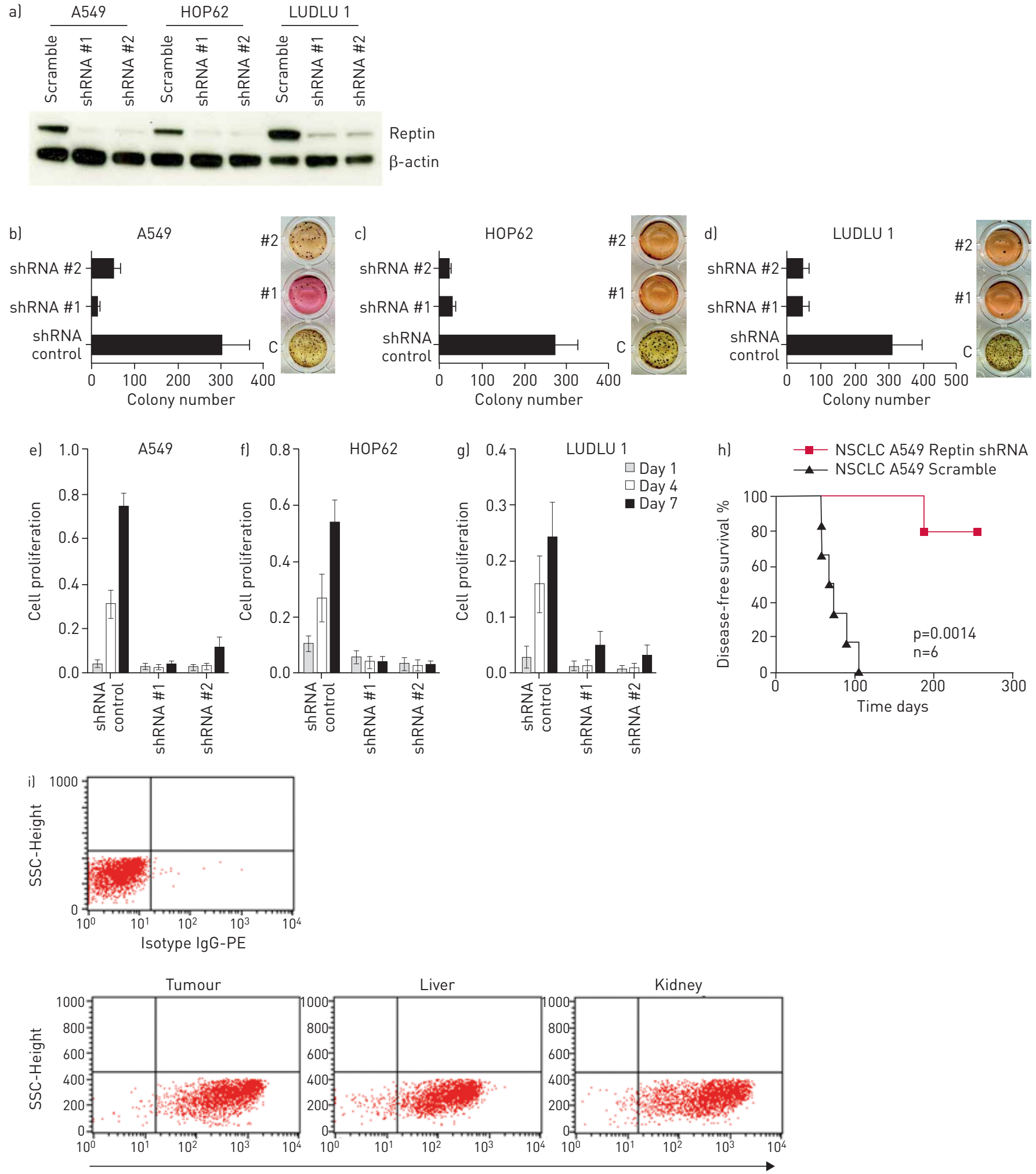

CD-326-PE

FIGURE 3 Knockdown of Reptin suppresses clonal expansion and proliferation of human nonsmall cell lung cancer (NSCLC) cells ex vivo and impairs engraftment in a xenograft model. a) Knockdown of Reptin in human pulmonary adenocarcinoma cells (A549 and HOP62) and squamous cell carcinoma (SCC) cells (LUDLU 1) via validated shRNAs \#1 and \#2 as compared to scrambled controls, assessed via immunoblotting. b-d) Colony numbers of A549, HOP62 and LUDLU 1 lung cancer cells, respectively, in methylcellulose upon knockdown of Reptin and as compared to scrambled controls (C) and e-g) proliferation of these cells as assessed by MTS assay. Error bars represent SD of at least three independent experiments. h) Disease-free survival of NSG mice injected with A549 NSCLC cells with shRNA-mediated knockdown of Reptin as compared to scramble control group. A log-rank (Mantel-Cox) test was used to compare survival curves. $p$-values $<0.05$ were considered statistically significant. i) Representative flow cytometry analysis after staining cells from metastatic tissues from mice of control group with human anti-CD-326-PE. Ig: immunoglobulin. 
a)

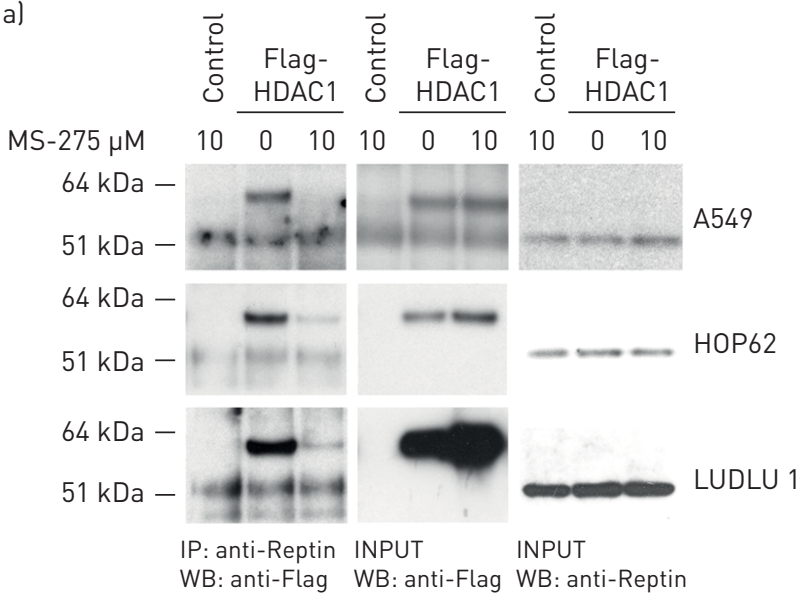

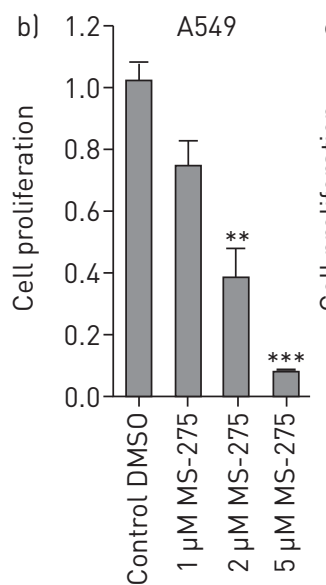

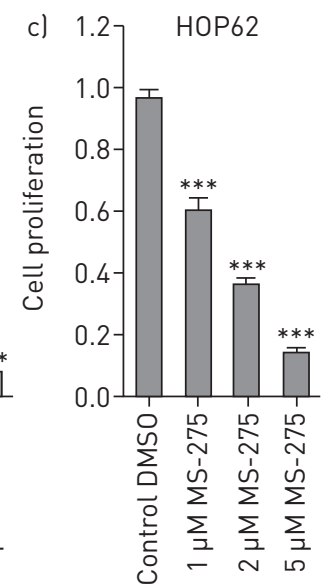

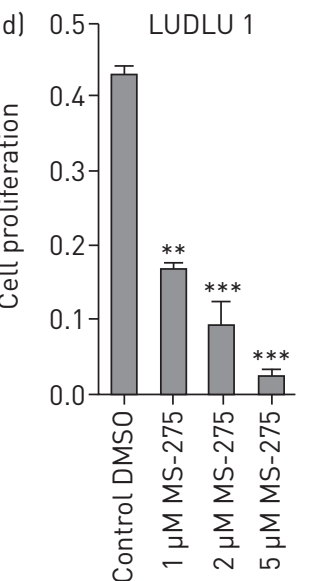
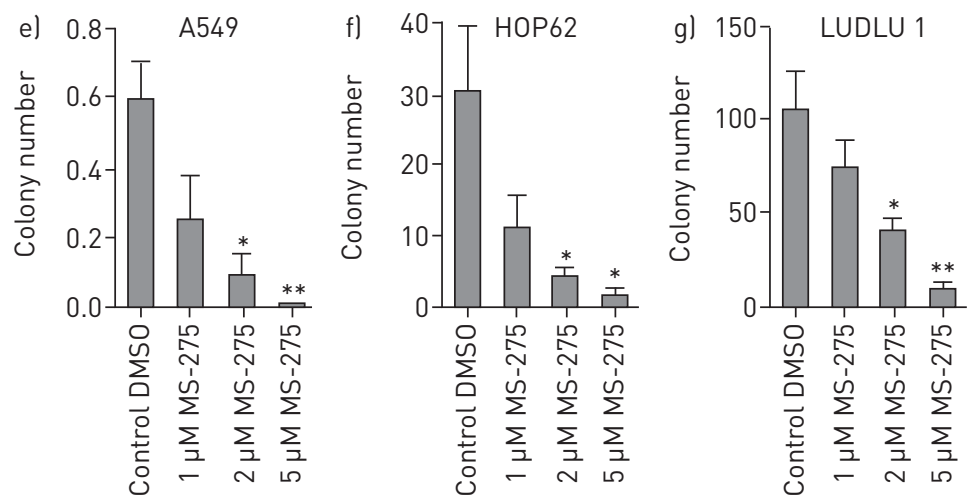

h)

MS-275 $\mu \mathrm{M}$

A549

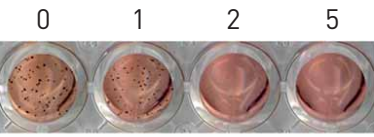

HOP62

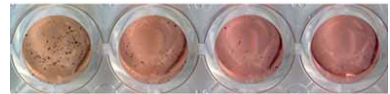

LUDLU 1

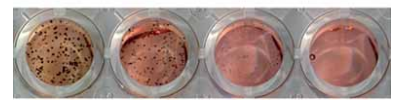

FIGURE 4 Pharmacological disruption of Reptin/HDAC1 complex significantly suppresses colony formation and proliferation of human nonsmall cell lung cancer (NSCLC) cells. a) Co-immunoprecipitation analyses of human A549, HOP62 and LUDLU 1 cells in the presence or absence of Flag-tagged HDAC1, and cultured in the presence or absence of MS-275 at the indicated concentration. b-d) Proliferation assays, e-g) colony formation assays and h) INT staining of the three indicated human NSCLC cell lines in the presence or absence of MS-275. Colonies were counted after 7 days. A one-way ANOVA test was used to determine statistical significance for all bar charts representing proliferation and colony formation assays of cells treated with MS-275. Error bars represent SD. DMSO: dimethyl sulfoxide. ${ }^{*}: p<0.05 ;{ }^{* *}: p<0.01 ;{ }^{* * *}: p<0.001$.

unveiling the key mechanisms that deregulate the transcriptional and epigenetic programmes driving lung cancer remains the main goal in lung cancer research.

We reveal a novel function of the AAA+ ATPase Reptin as a key player in NSCLC. We were able to show that Reptin is highly expressed in the vast majority of NSCLC tumours irrespective of their histological subtype and that high expression levels of Reptin are associated with an adverse prognosis for affected patients. Moreover, the comparably poor prognosis of NSCLC patients with well-differentiated tumours and high Reptin expression suggests that this protein is a predictor of early metastasis in this subgroup. We provide several lines of evidence that Reptin functions as a critical regulator of NSCLC cell proliferation and clonal growth. Importantly, knockdown of Reptin in NSCLC cells significantly impairs their capacity to induce metastatic lung cancer in a murine xenograft model.

Thus, our data present Reptin as a potential new molecular marker for the prediction of prognosis of NSCLC patients as well as a future therapeutic target for NSCLC treatment.

However, direct targeting of Reptin is not yet applicable. Thus, targeting key interaction partners of Reptin could be a reasonable alternative approach to impair signalling pathways directed by Reptin. The fact that Reptin can be part of different multi-protein complexes implicates its function in a variety of cellular processes, including those relevant for oncogenesis and tumour progression. A Reptin chromatin remodelling complex directing transcriptional regulation in coordinated action with beta-catenin has been shown to downregulate KAI1 [17]. However, knockdown of Reptin in NSCLC cells did not affect expression levels of KAI1 (data not shown). Thus, our data implicate a novel pathway driven via a Reptin/HDAC1 interaction specifically in NSCLC. Interestingly, high expression of HDAC1 confers poor prognosis in patients with AC lung cancer [15]. In our study, we were able to show that in NSCLC cells 

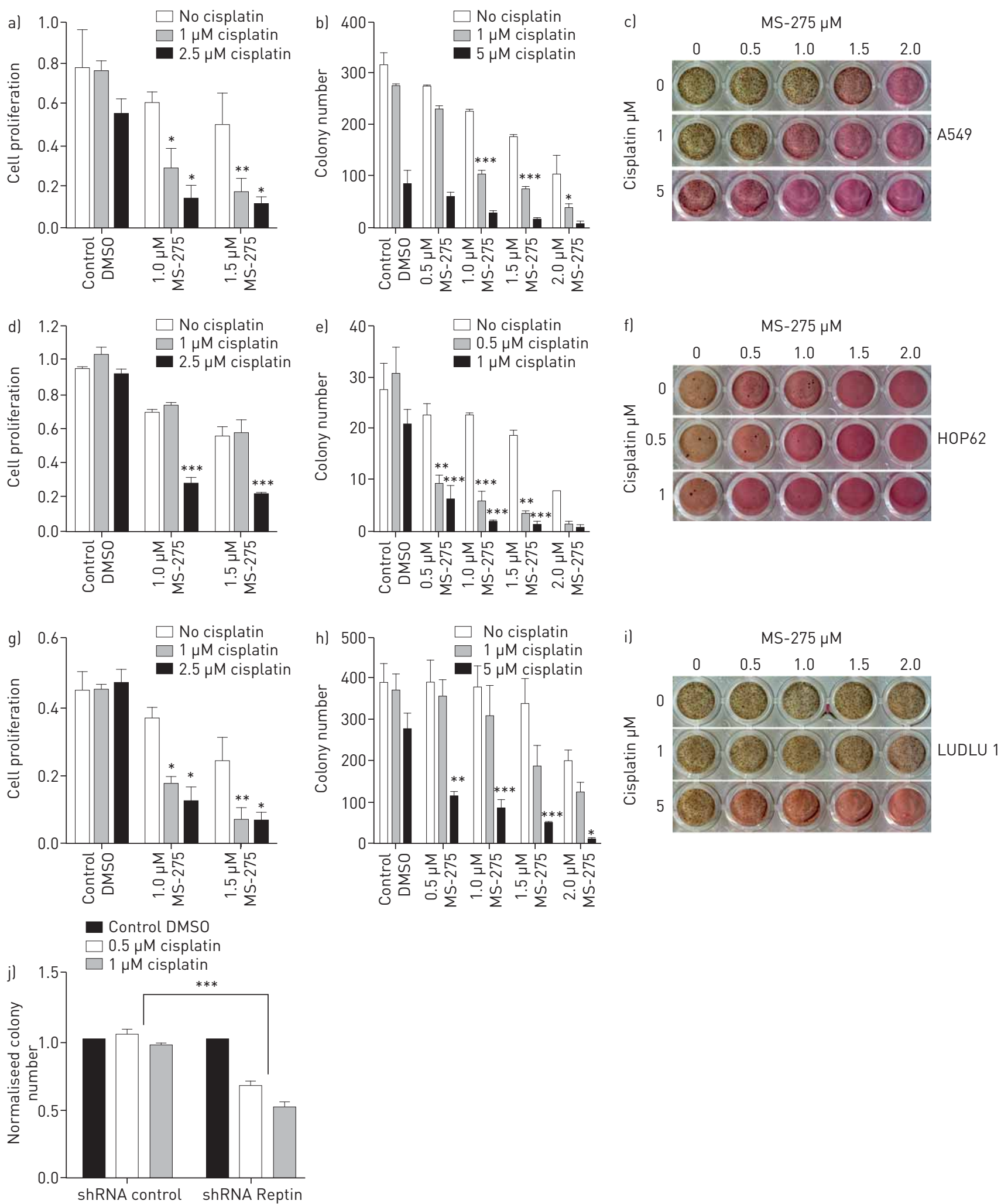

FIGURE 5 Disruption of Reptin/HDAC1 protein complex by MS-275 sensitises nonsmall cell lung cancer (NSCLC) cells to cisplatin. Combined treatment of human NSCLC cells of a-c) A549, d-f) HOP62 and g-i) LUDLU 1 using cisplatin and MS-275 at the indicated concentrations: proliferation assays $(a, d, g)$, colony formation assays $(b, e, h)$ and INT staining $(c, f, i)$ are displayed. A two-way ANOVA test was used to determine statistical significance for all bar charts for proliferation and colony formation assays of cells under combination treatment. j) Knockdown of Reptin sensitises human NSCLC cells to treatment with cisplatin. shRNA-mediated knockdown of Reptin enhances the effect of cisplatin treatment on clonal expansion of human A549 NSCLC cells ex vivo as compared to scramble control. Error bars represent SD of at least three independent experiments. DMSO: dimethyl sulfoxide. ${ }^{*}: p<0.05 ;{ }^{* *}: p<0.01{ }^{* * *}: p<0.001$. 
Reptin recruits HDAC1 into a protein complex, and that this complex can be disrupted upon exposure to MS-275, a potent HDAC1 inhibitor, resulting in substantial suppression of growth of lung cancer cells.

HDACs largely contribute to oncogenesis and cancer progression [18]. Therefore, inhibition of HDACs has become highly attractive for targeted cancer therapies. Multiple clinical trials using HDAC inhibitors have been concluded and are underway to assess the potential of these drugs in various types of cancer, and the first HDAC inhibitors have recently been approved for several malignant diseases. However, single-agent HDAC inhibition may not be feasible as a therapeutic approach for NSCLC treatment, given that various clinical trials including some for NSCLC have largely failed to show breakthrough effects using HDAC inhibitors as a monotherapy [19], and high doses of HDAC inhibitors confer considerable toxicity to patients [20]. Yet, results from various clinical trials suggest that combination treatments including HDAC inhibition are far more promising for oncological treatment strategies [19]. Thus, in our study we exposed pulmonary AC and SCC cells to low doses of MS-275 in combination with cisplatin. MS-275 significantly enhanced cell response to cisplatin, showing a substantial effect even at concentrations of cisplatin that do not have relevant efficacy as a single agent.

Because the majority of patients with locally advanced or metastatic NSCLC do not harbour oncogenic drivers directly tractable via specific inhibitors, they still receive platinum-based double chemotherapies in specific neoadjuvant, adjuvant and palliative treatment settings. Furthermore, many patients initially treated with molecular therapies will require chemotherapy at a later stage of their course of therapy. The substantial toxicity as well as the limited rate and duration of responses resulting from these cytotoxic regimens drive the need for new substances that improve treatment response and toxicity profiles. Previous lung cancer trials with combination treatments including HDAC inhibitors failed to significantly improve patient outcomes in most subgroups. However, the more specific and selective HDAC inhibitor MS-275 should still be considered a promising drug for NSCLC patients, even more so given that its effect appears to be more profound on transformed than on normal cells [21]. It may just need to be applied together with the right partner. The mechanisms of action of HDACs in NSCLC need to be precisely understood and their inhibition should be as targeted as possible. Here, as a proof of principle study, we were able to show that combined treatment with cisplatin and entinostat could be used successfully to significantly enhance human NSCLC cell responses to platinum-based therapy. In order to reveal the spectrum of cellular mechanisms affected by this combined treatment, future proteomic and epigenetic analyses that focus on Reptin/HDAC1 interactions might help to elucidate the dynamics of signalling pathways driven by this complex and its inhibition in NSCLC cells.

In conclusion, our results describe Reptin as a novel regulator that mediates the proliferation and clonal growth of human NSCLC cells ex vivo and in vivo. We show that high Reptin expression confers poor prognosis in lung cancer patients and we present a Reptin/HDAC1 protein complex whose pharmacological disruption sensitises NSCLC cells to cisplatin. These results shed light onto NSCLC oncogenesis and may help to identify patients at higher risk for early disease progression or relapse and those that are less likely to respond satisfactorily to platinum-based chemotherapies. Ultimately, our study may pave the way to new therapeutic strategies combining HDAC1 inhibitors with current standard therapy regimens.

Acknowledgements: We would like to thank I. Buchroth (Gerhard Domagk Institute for Pathology, University of Muenster, Muenster, Germany) for help with the experimental work, and K. Glaser (Abbott Laboratories, Chicago, IL, USA) and C. Abraham (Dept. of Molecular, Cellular, and Developmental Biology, University of Colorado at Boulder, CO, USA), who kindly provided us with plasmids containing HDAC1 and shRNAs targeting human Reptin, respectively.

Conflict of interest: None declared.

Support statement: Our work was generously funded by Else Kröner-Fresenius-Stiftung (No. 2015_A112). W.E. Berdel's laboratory is supported by Deutsche Forschungsgemeinschaft, DFG EXC 1003 Cells in Motion - Cluster of Excellence, Muenster, Germany. Funding information for this article has been deposited with the Crossref Funder Registry.

\section{References}

$1 \quad$ Heist RS, Engelman JA. SnapShot: non-small cell lung cancer. Cancer Cell 2012; 21: 448.

2 Hirsch FR, Herbst RS, Gandara DR. EGFR tyrosine kinase inhibitors in squamous cell lung cancer. Lancet Oncol 2015; 16: 872-873.

3 Kwak EL, Bang Y-J, Camidge DR, et al. Anaplastic lymphoma kinase inhibition in non-small-cell lung cancer. N Engl J Med 2010; 363: 1693-1703.

4 Reck M, Rodríguez-Abreu D, Robinson AG, et al. Pembrolizumab versus chemotherapy for PD-L1-positive non-small-cell lung cancer. N Engl J Med 2016: 1823-1833.

5 Allemani C, Weir HK, Carreira H, et al. Global surveillance of cancer survival 1995-2009: analysis of individual data for 25,676,887 patients from 279 population-based registries in 67 countries (CONCORD-2). Lancet 2015; 385: $977-1010$. 
6 Swanton C, Govindan R. Clinical implications of genomic discoveries in lung cancer. N Engl J Med 2016; 374 1864-1873.

7 Matias PM, Baek SH, Bandeiras TM, et al. The AAA+ proteins Pontin and Reptin enter adult age: from understanding their basic biology to the identification of selective inhibitors. Front Mol Biosci 2015; 2: 17.

$8 \quad$ Gallant P. Control of transcription by Pontin and Reptin. Trends Cell Biol 2007; 17: 187-192.

9 Schmidt LH, Kümmel A, Görlich D, et al. PD-1 and PD-L1 expression in NSCLC indicate a favorable prognosis in defined subgroups. PLoS One 2015; 10: e0136023.

10 Harvey JM, Clark GM, Osborne CK, et al. Estrogen receptor status by immunohistochemistry is superior to the ligand-binding assay for predicting response to adjuvant endocrine therapy in breast cancer. J Clin Oncol 1999; 17: 1474-1481.

11 Gallant-Behm CL, Ramsey MR, Bensard CL, et al. $\Delta \mathrm{Np} 63 \alpha$ represses anti-proliferative genes via $\mathrm{H} 2 \mathrm{~A}$. Z deposition. Genes Dev 2012; 26: 2325-2336.

12 Györffy B, Surowiak P, Budczies J, et al. Online survival analysis software to assess the prognostic value of biomarkers using transcriptomic data in non-small-cell lung cancer. PLoS One 2013; 8: e82241.

13 Pilotto S, Sperduti I, Novello S, et al. Risk stratification model for resected squamous-cell lung cancer patients according to clinical and pathological factors. J Thorac Oncol 2015; 10: 1341-1348.

14 Rashid S, Pilecka I, Torun A, et al. Endosomal adaptor proteins APPL1 and APPL2 are novel activators of beta-catenin/TCF-mediated transcription. J Biol Chem 2009; 284: 18115-18128.

15 Minamiya $\mathrm{Y}$, Ono T, Saito H, et al. Expression of histone deacetylase 1 correlates with a poor prognosis in patients with adenocarcinoma of the lung. Lung Cancer 2011; 74: 300-304.

16 Reck M, Krzakowski M, Chmielowska E, et al. A randomized, double-blind, placebo-controlled phase 2 study of tigatuzumab (CS-1008) in combination with carboplatin/paclitaxel in patients with chemotherapy-naïve metastatic/unresectable non-small cell lung cancer. Lung Cancer 2013; 82: 441-448.

17 Kim JH, Kim B, Cai L, et al. Transcriptional regulation of a metastasis suppressor gene by Tip60 and beta-catenin complexes. Nature 2005; 434: 921-926.

18 Falkenberg KJ, Johnstone RW. Histone deacetylases and their inhibitors in cancer, neurological diseases and immune disorders. Nat Rev Drug Discov 2014; 13: 673-691.

19 New M, Olzscha H, La Thangue NB. HDAC inhibitor-based therapies: can we interpret the code? Mol Oncol 2012; 6: 637-656.

20 Subramanian S, Bates SE, Wright JJ, et al. Clinical toxicities of histone deacetylase inhibitors. Pharmaceuticals (Basel) 2010; 3: 2751-2767.

21 Ungerstedt JS, Sowa Y, Xu W-S, et al. Role of thioredoxin in the response of normal and transformed cells to histone deacetylase inhibitors. Proc Natl Acad Sci USA 2005; 102: 673-678. 